\title{
The Effect of Phloroglucinol in Patients With Diarrhea-predominant Irritable Bowel Syndrome: A Randomized, Double-blind, Placebo-controlled Trial
}

\author{
Seung Yong Shin, ${ }^{1}$ Bong Ki Cha, ${ }^{2}$ Won-Seok Kim, ${ }^{1}$ Jae Yong Park, ${ }^{1}$ Jeong Wook Kim, ${ }^{1}$ and Chang Hwan Choi ${ }^{1 *}$ \\ ${ }^{\prime}$ Department of Internal Medicine, Chung-Ang University College of Medicine, Seoul, Korea; and ${ }^{2}$ Department of Internal Medicine, Chung-Ang \\ Medical Health Care System Hyundae Hospital, Seoul, Korea
}

\section{Background/Aims}

We aim to evaluate the efficacy and safety of phloroglucinol in patients with diarrhea-predominant irritable bowel syndrome (IBS-D).

\section{Methods}

Seventy-two patients with IBS-D who met Rome III criteria were 1:1 randomized in a parallel, double-blind design to receive phloroglucinol or placebo for 2 weeks. Patients were followed for 1 week after the end of treatment. The primary outcome was the proportion of responders, defined as those who answered "moderate or more of improvement" to the subject global assessment for at least 1 week of the 2-week treatment period. Secondary outcomes included the proportion of these patients during the 3-week period including 1 week of follow-up, IBS symptoms (abdominal pain/discomfort, diarrhea, urgency, mucus in stool, bloating, and passage of gas), stool frequency and consistency, and IBS quality of life (IBS-QOL).

\section{Results}

The proportion of responders during 2-week treatment period tended to be higher in the phloroglucinol group than in the placebo group, although the difference did not reach statistical significance (55.6\% vs $30.6 \%, P=0.056)$. The proportion of responders during the 3-week period was significantly higher in the phloroglucinol group than in the placebo group $(61.6 \%$ vs $30.6 \%, P=0.013$ ). Individual symptom scores, IBS-QOL, stool frequency and consistency tended to improve in the phloroglucinol group, but there were no statistical significances compared to those of the placebo group. No serious adverse events were reported in both groups.

\section{Conclusions}

Phloroglucinol could be a safe and beneficial option for the management of overall IBS symptoms in patients with IBS-D. Further large scaled studies are warranted.

(J Neurogastroenterol Motil 2020;26:117-127)

\section{Key Words}

Antispasmodic agent; Irritable bowel syndrome; Phloroglucinol

\footnotetext{
Received: August 20, 2019 Revised: October 24, 2019 Accepted: November 8, 2019

(a) This is an Open Access article distributed under the terms of the Creative Commons Attribution Non-Commercial License (http://creativecommons. org/licenses/by-nc/4.0) which permits unrestricted non-commercial use, distribution, and reproduction in any medium, provided the original work is properly cited.

*Correspondence: Chang Hwan Choi, MD, PhD Department of Internal Medicine, Chung-Ang University College of Medicine, 102 Heukseok ro, Dongjak gu, Seoul 06973, Korea Tel: +82-2-6299-1418, Fax: +82-2-6299-2017, E-mail: gicch@cau.ac.kr

Seung Yong Shin and Bong Ki Cha contributed equally to this work
} 


\section{Introduction}

Irritable bowel syndrome (IBS) is a relatively common functional gastrointestinal (GI) disorder characterized by recurrent abdominal pain related to defecation or in association with a change in bowel habits. ${ }^{1}$ IBS is a chronic disorder that may lead to impaired personal and social interactions of affected individuals., ${ }^{2,3}$ However, the pathophysiology of IBS is still unclear. Various factors including visceral hypersensitivity, abnormal gut motility, immune activation, disorder of the brain-gut axis, impaired intestinal barrier function, and alteration of gut microbiota, have been suggested as main causes of IBS in previous literatures. ${ }^{4-6}$

Although the potential causes of IBS may overlap in an individual and vary in importance between patients, increased sensitivity to pain and altered intestinal motility are the main factors that contribute to IBS symptoms, especially abdominal discomfort and diarrhea. ${ }^{3,7,8}$ Previous studies have reported an association between pain episodes and the motor activity of jejunum. ${ }^{9-11}$ Abnormal colonic motility has also been suggested to provoke abdominal pain in patients with IBS. ${ }^{7,812}$ Based on these results, antispasmodics have been widely used for symptomatic relief in IBS patients. Antispasmodics, a group of drugs which directly affect the smooth muscle or cholinergic receptors, are believed to reduce IBS-related pain by the inhibition of intestinal contractile pathways. Additionally, they improve bowel habits by decreasing colonic transit and, thereby, reduce bowel movement. ${ }^{8,13}$ Previous meta-analyses have shown that antispasmodics provide significant benefit in abdominal pain and improve overall IBS symptom. ${ }^{14,15}$ However, a number of reviews have reported that the efficacy of antispasmodics are questionable in patients with IBS because most studies were conducted decades ago and did not meet the current requirements for high-quality trials in IBS. ${ }^{16-18}$ Phloroglucinol, a phenol derivative with non-specific antispasmodic effect, acts directly on the smooth muscle. Phloroglucinol leads to muscle relaxation by inhibiting voltage-dependent calcium channels ${ }^{19,20}$ and has none of the anticholinergic side effects associated with the other classes of antispasmodics. ${ }^{14,15,20}$ In a previous study, phloroglucinol reduced motility of rectosigmoid following a test meal ${ }^{21}$ and inhibited colonic phasic contractions promoted by glycerol injection into rectum without affecting colonic tone. ${ }^{22}$ However, previous data regarding the efficacy and safety of phloroglucinol in patients with IBS are very limited despite its widespread use. Therefore, we aim to evaluate the efficacy and safety of phloroglucinol in patients with diarrhea-predominant IBS (IBS-D) in a randomized, double-blind, placebo-controlled trial.

\section{Materials and Methods}

\section{Patients}

Patients were enrolled from the gastroenterology clinic from September 2009 until October 2010. Based on the inclusion and exclusion criteria, experienced gastroenterologists evaluated patients for eligibility at a screening visit. Patients between the ages of 18 years and 65 years who met the Rome III criteria for diagnosis of IBS-D and who showed normal colonoscopy findings within the previous 5 years were eligible in the study. Patients with the following clinical features were excluded: hypersensitivity to phloroglucin$\mathrm{ol}$, its derivatives, and other antispasmodics; pregnancy or lactation during the study period; history of drug or alcohol abuse 6 months prior to screening; abnormal results from screening laboratory tests with clinical relevance for study participation; severe medical disorders (liver disease, heart disease, renal disease, endocrine disorder, neurological disease, and malignant tumors); other GI diseases that may explain the patient's symptoms, as judged by the researcher; symptoms that may indicate other severe diseases, such as GI bleeding, weight loss, or fever; history of glaucoma or benign prostate hyperplasia; history of psychiatric disorder; history of other abdominal surgery, except appendectomy and operation for hernias; consumption of corticosteroids, nonsteroid anti-inflammatory drugs, or other anti-inflammatory drugs 2 weeks prior to screening and throughout the study; use of drugs that may influence efficacy evaluations, such as probiotics, antibiotics, prokinetics, antispasmodics, or antidepressants 2 weeks prior to screening and throughout the study; participation in other clinical trial within 3 months before the start of this trial; or judged ineligible by the investigators. All patients were able to understand the study protocols, and written informed content was obtained from each patient prior to commencement of the study.

\section{Study Protocol}

A randomized, double-blind, placebo-controlled clinical trial was conducted. Figure 1 summarizes the overall study design. During the 1-week screening period, we evaluated each patient based on a full review of personal medical history and physical examination; further, a complete blood count and serum chemistry were performed. Patients with clinically serious abnormalities in any of the results were excluded from randomization. Eligible patients who met the inclusion and exclusion criteria received questionnaires and were asked to complete a symptom diary and IBS quality of 


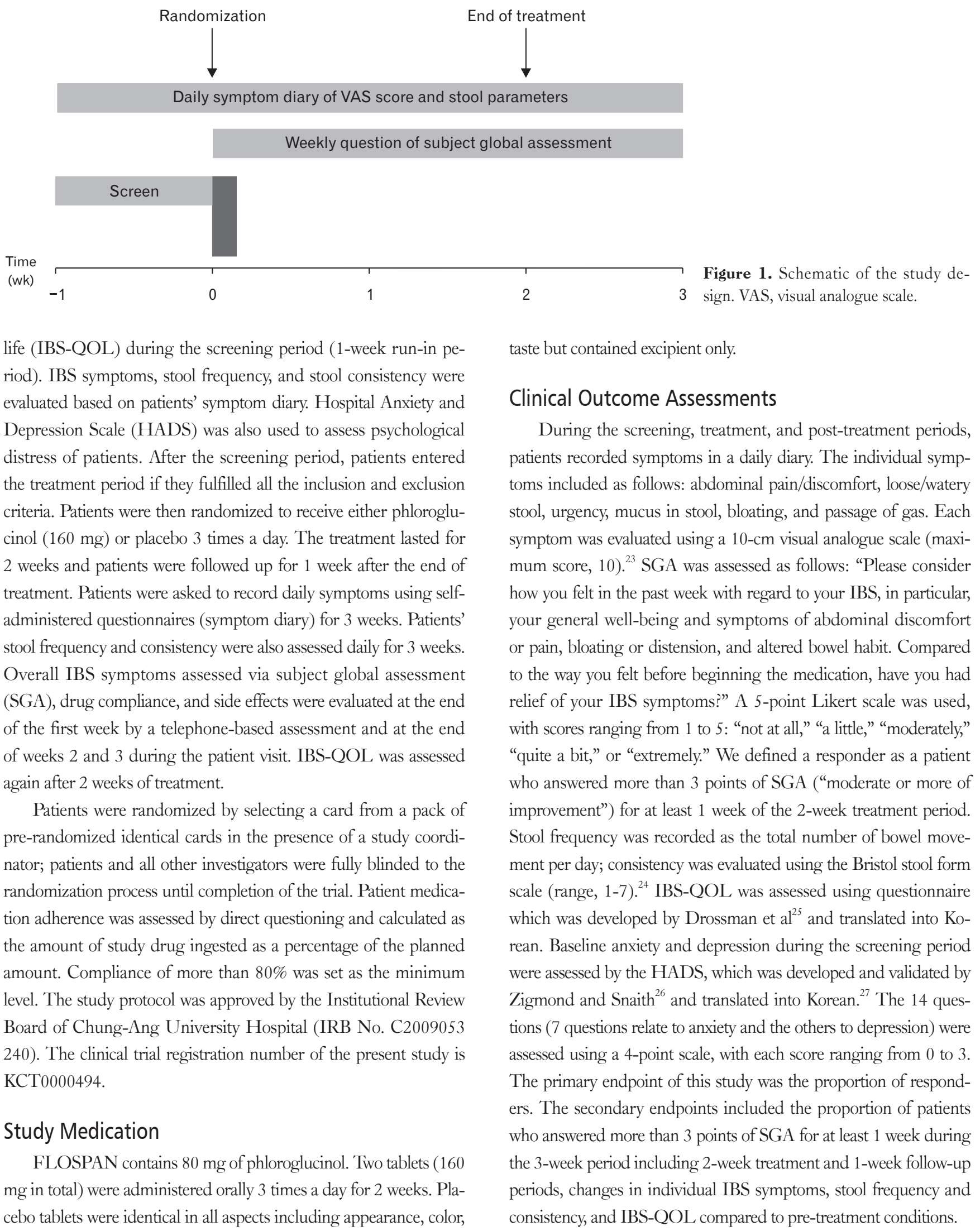




\section{Safety Assessments}

Adverse events related to treatment were actively monitored throughout the study period. Drug-related adverse events were assessed by direct questioning at each week. Either physical examination and routine laboratory tests (complete blood count and serum chemistry) were performed at the screening period and early withdrawal. Patients' vital signs including body temperature, respiratory rate, pulse, and blood pressure were monitored by investigators at each visit. Safety assessments included adverse events, abnormal laboratory findings, and vital signs from the screening period to study completion.

\section{Statistical Methods}

All data collections and analyses were performed independently from the investigators who had no access to data or analyses until the latter had been completed. An imputation method was applied for missing data or loss to follow-up.

SPSS for Windows, version 18.0 (IBM Corp, Armonk, NY, USA), was used for all data analyses. For categorical variables, a Pearson Chi-square test was used. Student $t$ test was used to compare the means of continuous variables, and continuous variables are presented as the mean \pm standard deviation. The two-sided $P$ values $<0.05$ were considered statistically significant.

The planned sample size for this study $(n=72)$ was based on the ability to detect a $25 \%$ difference in the proportion of responders between the 2 groups, with $80 \%$ power at $\alpha=0.05$. Further, it was increased to account for a drop-out rate of just above $10 \%$.

\section{Results}

\section{Baseline Characteristics}

In total, 72 patients were screened from September 2009 to October 2010. All patients were randomized and entered the treatment period. Among the 72 evaluable subjects, 26 (36.1\%) were male and $46(63.9 \%)$ were female. The subjects averaged mean 42.3 years in age (range, 19-65 years). Thirty-six patients were assigned to the phloroglucinol group and 36 to the placebo group. No significant differences in terms of age, sex, body mass index, smoking, alcohol intake, or HADS scores were observed between the 2 groups. There were no differences in the severity of baseline IBS symptoms between the 2 groups (Table 1).

\section{Irritable Bowel Syndrome Symptoms and Stool Parameters}

During the study period, 5 patients assigned to the placebo group dropped out of the study. Of these, 3 patients withdrew their consent to participate in the study for personal reasons. The other 2 patients complained of nausea and abdominal pain and wished to discontinue the drug. Finally, a total of 67 patients satisfactorily completed the study (Fig. 2).

The proportion of responders who responded at least 1 week of the 2-week treatment period for overall IBS symptoms, tended to be higher in the phloroglucinol group than in the placebo group although the difference was not statistically significant $(55.6 \%$ vs $30.6 \%, P=0.056)$. The proportion of patients who answered more than 3 points of SGA for at least 1 week among the 3 weeks treatment and follow-up period was significantly higher in the phloroglucinol group than in the placebo group $(61.6 \%$ vs $30.6 \%$, $P=0.013)$. The proportions of patients who answered more than 3 points of SGA at each week also tended to be higher in the phloroglucinol group than in the placebo group, although the difference was not statistically significant (Week 1, 33.3\% vs $16.7 \%, P$ $=0.102$; Week $2,50.0 \%$ vs $30.6 \%, P=0.093$; Week $3,33.3 \%$ vs $13.9 \%, P=0.052$; Fig. 3 ).

Table 1. Baseline Characteristics and Irritable Bowel Syndromesymptoms of the Patients

\begin{tabular}{lccc}
\hline \multicolumn{1}{c}{ Characteristics } & $\begin{array}{c}\text { Phloroglucinol } \\
(\mathrm{n}=36)\end{array}$ & $\begin{array}{c}\text { Placebo } \\
(\mathrm{n}=36)\end{array}$ & $P$-value \\
\hline Age $(\mathrm{yr})$ & $42.30 \pm 12.77$ & $42.32 \pm 12.80$ & 0.993 \\
Sex & $11(30.6)$ & $15(41.7)$ & 0.156 \\
$\quad$ Male & $25(69.4)$ & $21(58.3)$ & \\
$\quad$ Female & $21.50 \pm 2.94$ & $22.66 \pm 3.10$ & 0.109 \\
BMI $\left(\mathrm{kg} / \mathrm{m}^{2}\right)$ & $3(8.3)$ & $3(8.3)$ & 0.622 \\
Smoker & $26(72.2)$ & $22(61.1)$ & 0.402 \\
Alcohol intake & $7.37 \pm 4.02$ & $7.91 \pm 5.41$ & 0.642 \\
Anxiety & $7.14 \pm 3.55$ & $7.70 \pm 4.86$ & 0.595 \\
Depression & & & \\
IBS symptoms & $4.42 \pm 2.22$ & $4.14 \pm 2.03$ & 0.597 \\
$\quad$ Abdominal pain/ & & & \\
$\quad$ discomfort & $4.31 \pm 2.75$ & $3.39 \pm 2.15$ & 0.126 \\
Loose/watery stool & $3.56 \pm 2.56$ & $3.06 \pm 1.94$ & 0.364 \\
$\quad$ Urgency & $1.47 \pm 2.00$ & $0.77 \pm 0.98$ & 0.069 \\
Mucus in stool & $4.36 \pm 2.53$ & $3.68 \pm 2.66$ & 0.288 \\
Bloating & $4.80 \pm 2.12$ & $3.98 \pm 2.01$ & 0.106 \\
\hline Passage of gas & & &
\end{tabular}

BMI, body mass index; IBS, irritable bowel syndrome.

Data are shown as mean $\pm \mathrm{SD}$ or $\mathrm{n}(\%)$. 
Figures 4 and 5 shows the changes of individual symptoms and stool parameters. Most of individual symptoms associated with IBS-D are improved in patients treated with phloroglucinol. Compared to the screening period, abdominal pain/discomfort, loose/watery stool, stool urgency, mucus in stool, passage of gas, and stool frequency and consistency were significantly improved after 2 weeks use of phloroglucinol. However, percent changes in individual symptom scores after 2 weeks were not different between the phloroglucinol group and placebo group. Stool frequency and consistency showed more decreasing tendencies in the phloroglucinol group compared to the placebo group, but the difference did not reach statistical significance (Table 2).

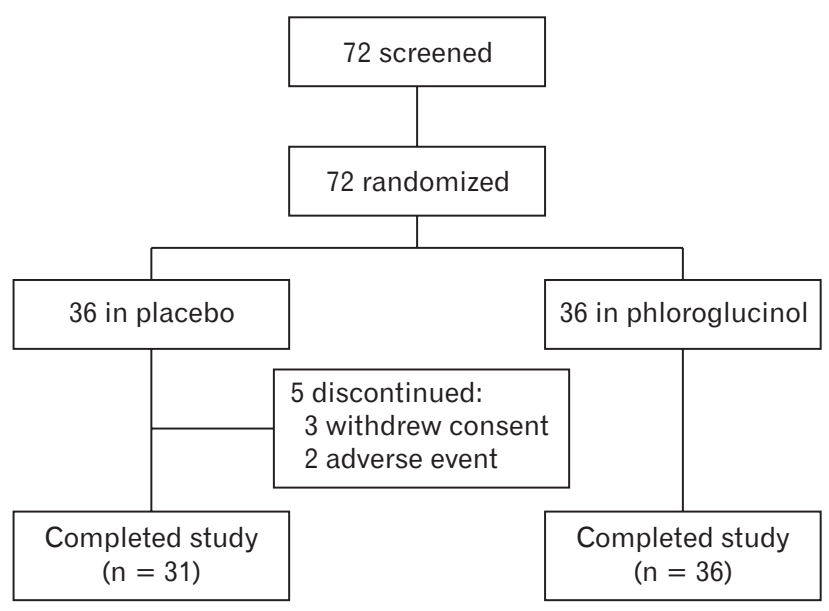

Figure 2. Flow chart showing patient disposition throughout the trial.

\section{Irritable Bowel Syndrome Quality of Life}

The mean of overall IBS-QOL score and that of several domains including interference with activity, health worry, and food avoidance significantly improved in the phloroglucinol group. The percentage change of overall IBS-QOL score tended to be higher in the phloroglucinol group compared to the placebo group, but the difference was not statistically significant $(17.03 \pm 41.33$ vs $6.96 \pm$ $26.45, P=0.252)$. Each domain score also showed more increasing tendency in the phloroglucinol group than the placebo group without statistical significance (Table 3).

\section{Safety and Tolerability}

The incidence of adverse events was $5.6 \%$ in both the placebo $(2 / 36)$ and phloroglucinol groups $(2 / 36)$. Two patients in the placebo group complained of nausea and abdominal discomfort and discontinued the study. Two patients in the phloroglucinol group complained of mild nausea but completed the study. No symptoms related to anticholinergic activity (such as dry mouth, dizziness, blurry vision, confusion, urinary retention, and constipation) were reported during the study period.

\section{Discussion}

The present study evaluated the efficacy and safety of phloroglucinol, compared with a placebo, in patients with IBS-D. The proportion of patients who experienced "moderate or more of improvement" of overall IBS symptoms for at least 1 week during the
A Responders for 2 weeks

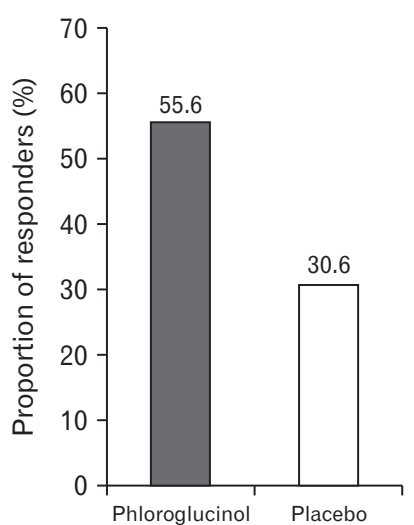

B Responders for 3 weeks

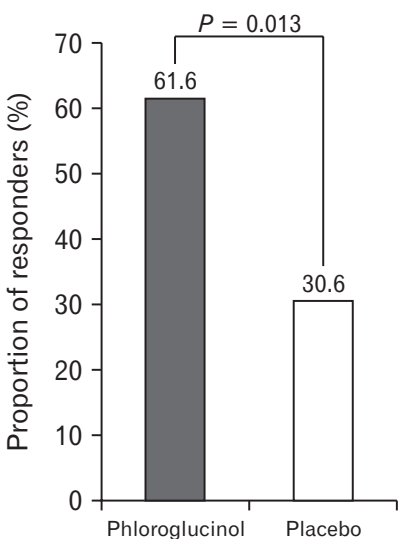

C Patients who answered more than 3 points of SGA at each week

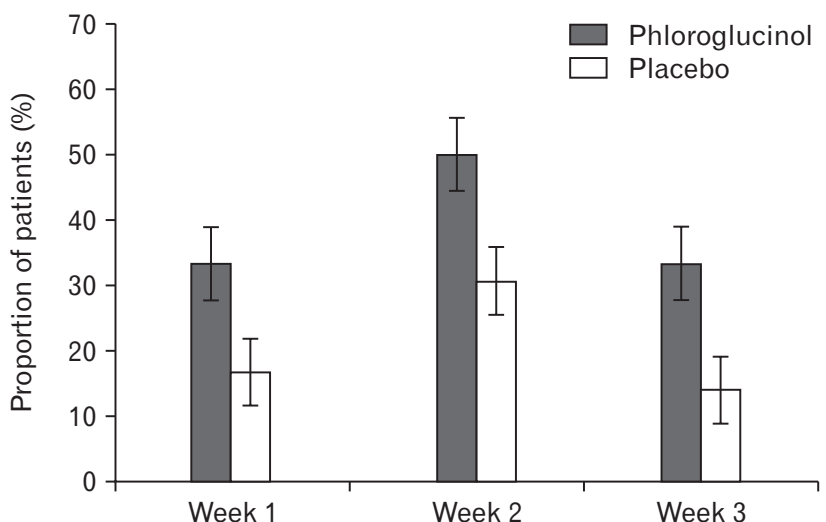

Figure 3. Proportion of patients who answered more than 3 points ("moderate or more of improvement") of subject global assessment (SGA) for at least 1 week of the 2-week treatment period (A), for at least 1 of the 3 weeks of treatment and follow-up period (B), and proportion of patients who answered more than 3 points of SGA at each week $(\mathrm{C})$ in the phloroglucinol and placebo groups. 
2-week treatment period tended to be higher in the phloroglucinol group than in the placebo group although this difference was not statistically significant $(P=0.056)$. The proportion of these pa-

A Abdominal pain/discomfort

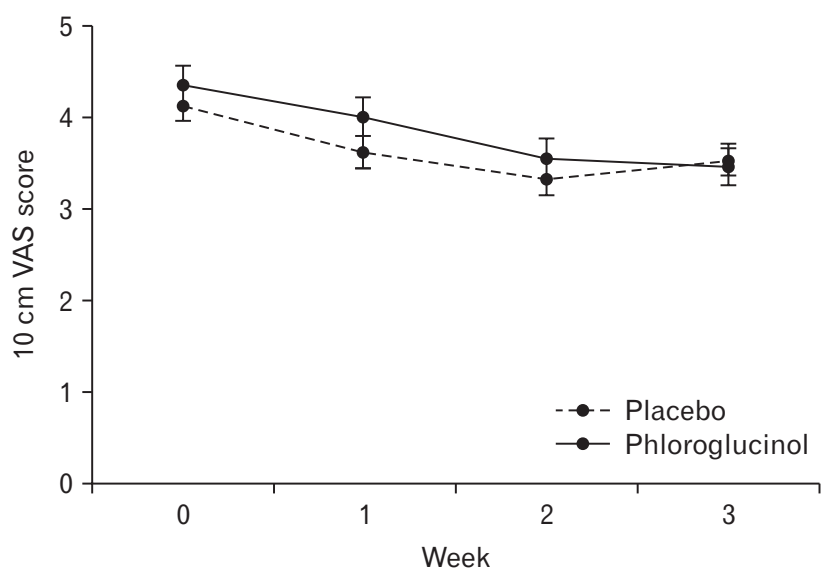

C Urgency

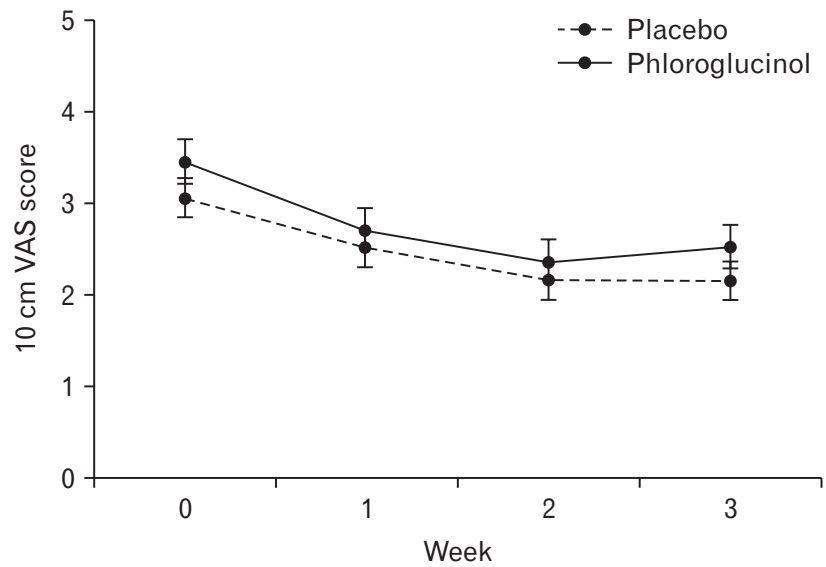

E Bloating

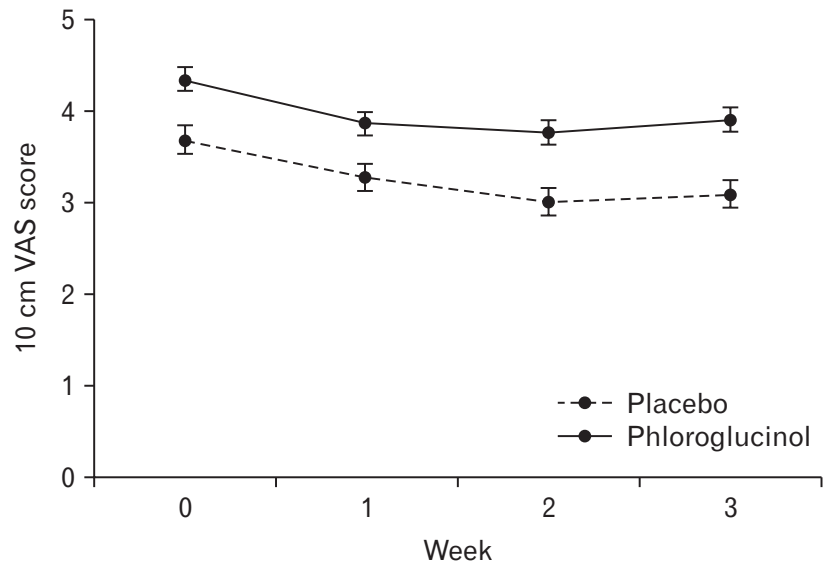

tients during the 3-week period including 1-week follow-up period was significantly higher in the phloroglucinol group than in the placebo group $(P=0.013)$. In terms of individual IBS symptoms,

B Loose/watery stool

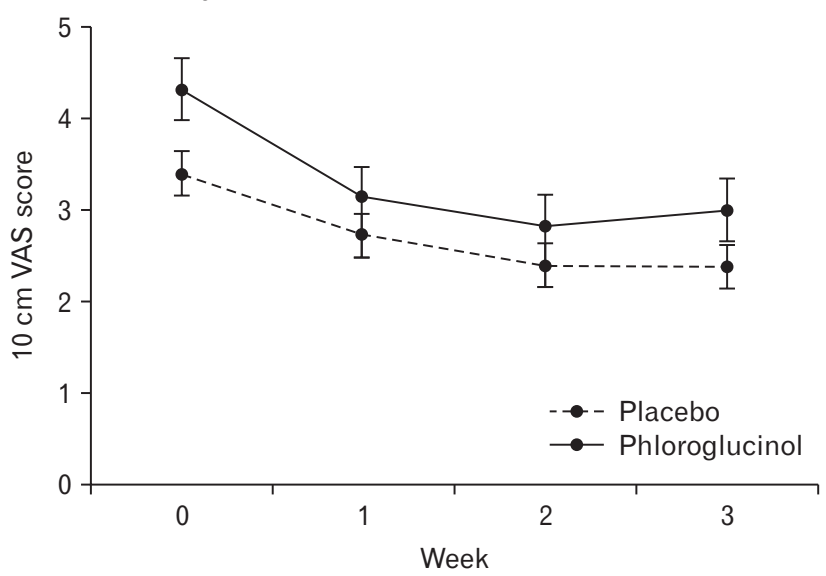

D Mucus in stool

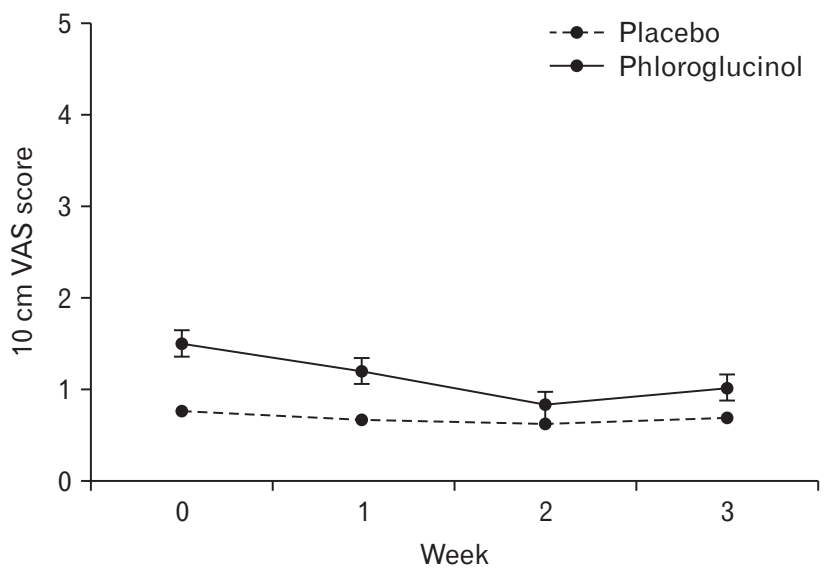

F Passage of gas

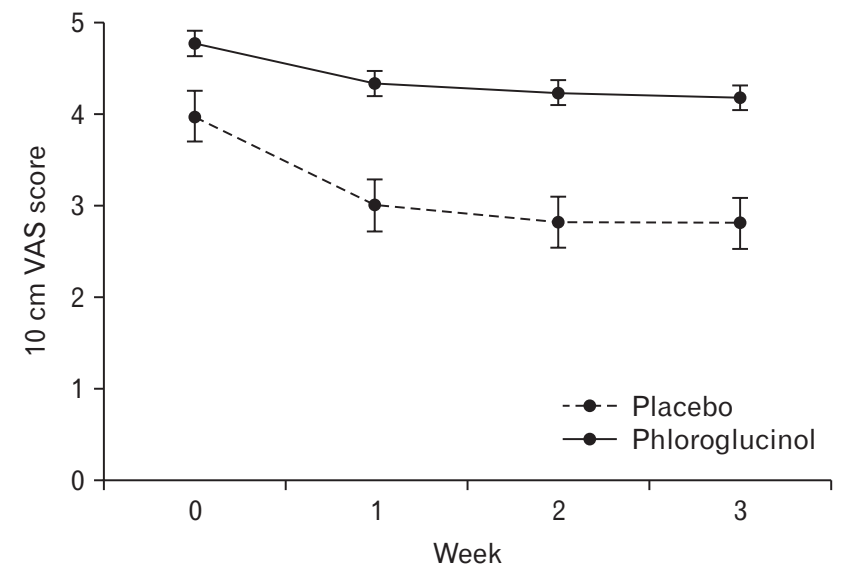

Figure 4. Symptoms associated with diarrhea-predominant irritable bowel syndrome. Changes of individual symptoms including abdominal pain/ discomfort (A), loose/watery stool (B), urgency (C), mucus in stool (D), bloating (E), and passage of gas (F) during study period. VAS, visual analogue scale. 

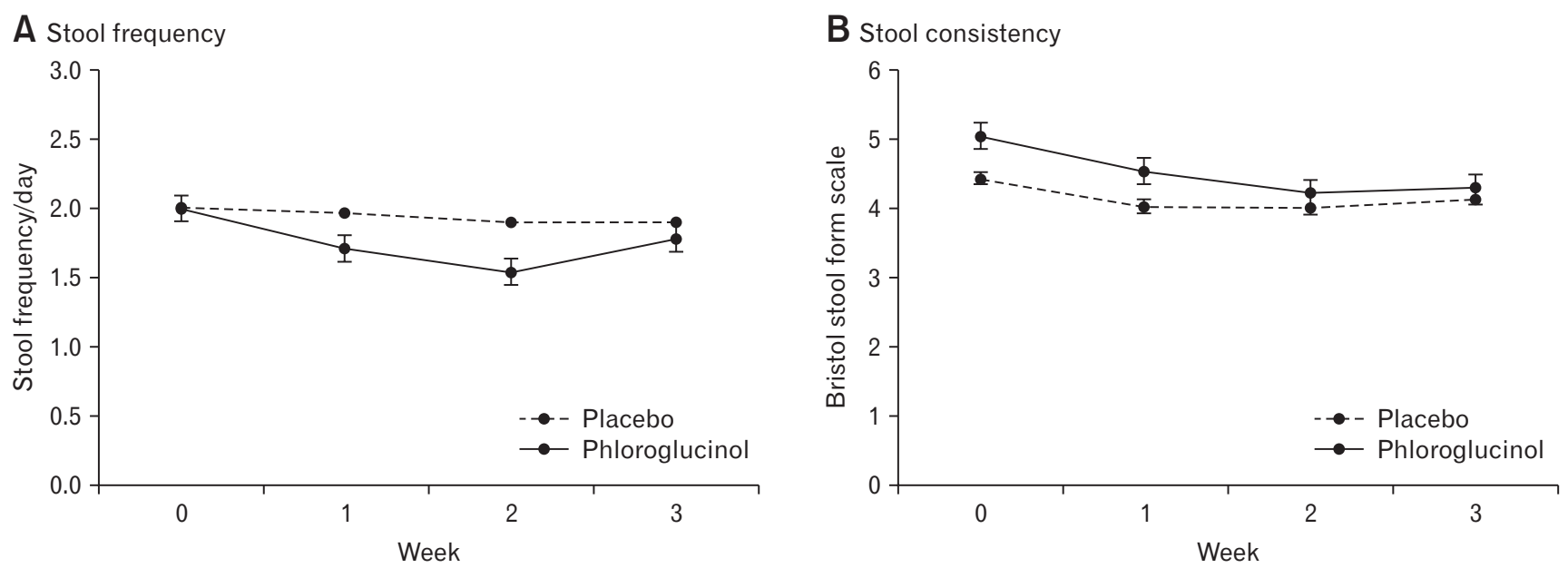

Figure 5. Changes of stool frequency (A) and consistency (B) during the study period.

including abdominal pain/discomfort, loose/watery stool, urgency, mucus in stool, and passage of gas, there were no significant differences in improvements between the 2 groups.

Traditional IBS therapies target the relief of individual IBS symptoms. However, they often demonstrate limited efficacy against overall IBS symptoms. ${ }^{28}$ Symptom-based pharmacologic therapies include antispasmodics, serotonin type 3 receptor antagonists, anti-diarrheals, tricyclic antidepressants, selective serotonin reuptake inhibitors, some probiotics, and antibiotics. Among these, antispasmodics are considered an effective treatment modality to improve abdominal cramps and reduce the stool frequency in patients with IBS because abdominal pain and discomfort are believed to be associated with intestinal motor dysfunction. Antispasmodics include broad class of drugs as follows; direct smooth muscle relaxants (eg, mebeverine and papaverine), drugs that directly act on calcium channels which affect intestinal smooth muscle contraction (eg, alverine citrate, otilonium bromide, and pinaverium bromide), and anticholinergic/antimuscarinic agents (eg, butylscopolamine, cimetropium bromide, and pirenzepine). ${ }^{20,29}$

The efficacy of antispasmodics has been reported to vary depending on the type of agent used. A Cochrane meta-analysis of 13 randomized controlled trials of antispasmodics, other than phloroglucinol, including 1392 patients with IBS, demonstrated a beneficial effect of these drugs on abdominal pain, with a pooled risk ratio of 1.32 (95\% CI, 1.12-1.55; $P<0.05)$. $^{15}$ Another meta-analysis of 23 double-blind randomized trials, including 1852 patients with IBS, demonstrated improved global assessment (56.0\% vs $38.0 \%$, 95\% CI, 1.77-2.58; $P<0.05)$ and pain relief $(53.0 \%$ vs $41.0 \%$, 95\% CI, 1.30-2.10; $P<0.05)$ in patients treated with a smooth muscle relaxant. ${ }^{30}$ Other meta-analyses on individual antispasmod- ics demonstrated that only pinaverium bromide and trimebutine had a significant benefit on abdominal pain. Other antispasmodics, including scopolamine derivates, dicyclomine, otilonium, and mebeverine, failed to show any benefit because of variable patient inclusion criteria, poorly designed studies, and significant heterogeneity among the studies. ${ }^{31}$ In a recent multi-center randomized controlled trial, including 356 IBS patients, otilonium bromide was found to be superior to a placebo in reducing the frequency of abdominal pain episodes at the end of the treatment period $(P<0.05)$. $^{32}$ However, the intensity of abdominal pain, the proportion of patients who responded did not differ between the 2 groups. Another multicenter, randomized controlled, non-inferiority trial demonstrated both tiropramide and otilonium significantly decreased abdominal pain and discomfort in 356 patients with IBS $(P<0.05)$; however, the change from baseline was not different between groups. ${ }^{33}$

Although limited data are available for phloroglucinol, the findings of previous studies demonstrate the efficacy of phloroglucinol in IBS patients. The intensity of pain during acute exacerbation of IBS was significantly reduced over a 1 -week period of oral phloroglucinol/trimethylphloroglucinol. ${ }^{34}$ Another open-label study reported that phloroglucinol significantly relieved IBS-related symptoms, including abdominal pain, bloating, stool frequency, urgency, and passage of mucus per rectum. ${ }^{35}$ In our study, however, the change rates of individual symptoms were not different between the phloroglucinol group and placebo group. These findings are unable to fully support the efficacy of phloroglucinol in patients with IBS-D. In the IBS drug trial, it has been difficult to prove a significant effect on individual symptoms because of the high placebo effect. ${ }^{36,37}$ Several previous studies of IBS patients have shown significant effects on overall symptoms or QOL, but the 

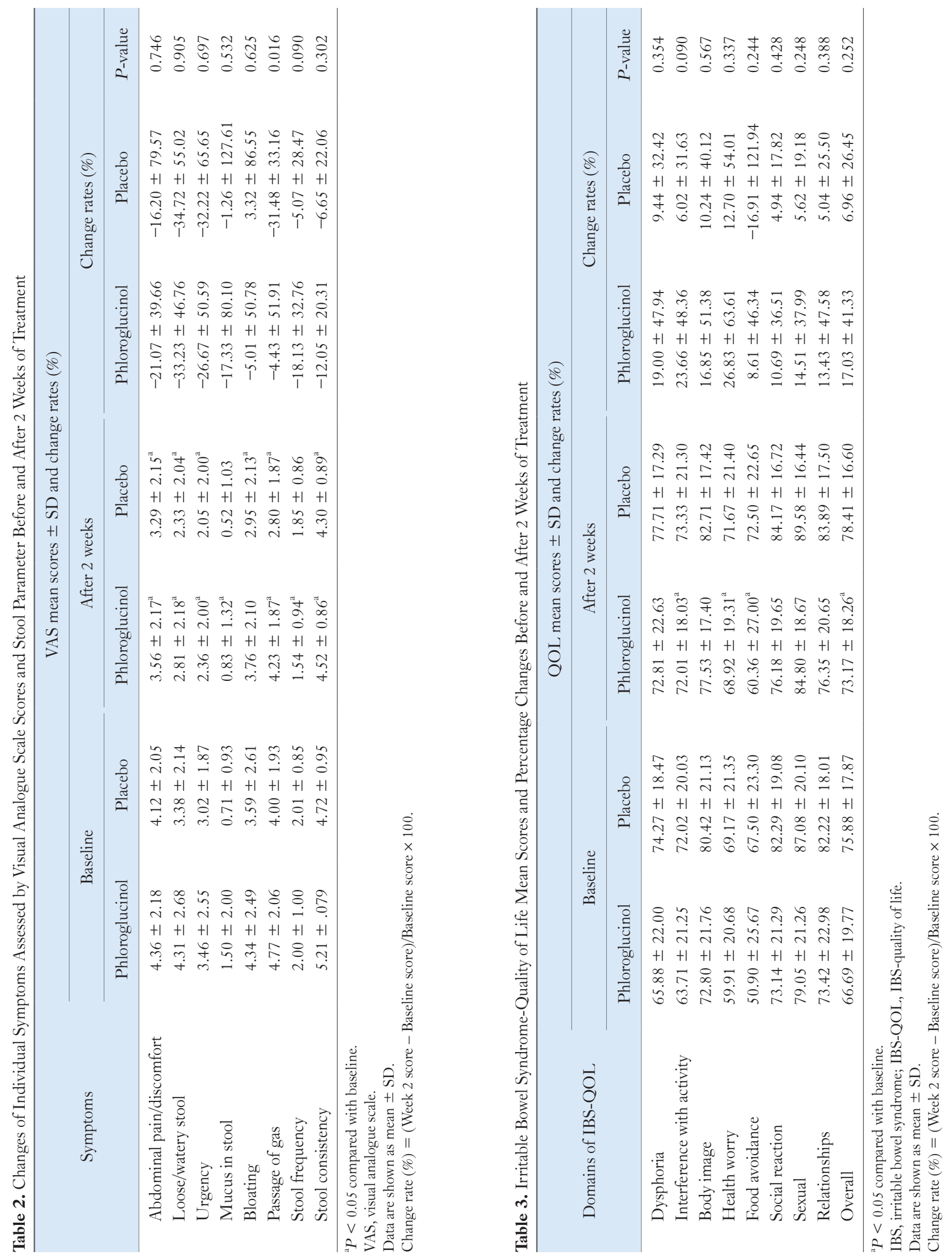
effects have been insufficient to improve individual symptoms. ${ }^{38-40}$ Also, the relatively small sample size may be related to these results. Further large scaled studies are needed to determine the effect of phloroglucinol in individual symptoms.

Antispasmodics have been used to relieve defecation symptoms due to their effects on reducing colonic transit and other, as yet unclear, pharmacological mechanisms. ${ }^{20}$ A non-inferiority study of tiropramide versus octilonium demonstrated that both agents improved stool parameters including stool frequency and consistency in a subgroup of patients with IBS-D. ${ }^{33}$ Phloroglucinol was also found to decrease phasic contractions by acting directly on the intestinal smooth muscle. ${ }^{35}$ This mechanism can decrease the hyperactive motility of the colon, and the accelerated colonic transit. In our study, stool frequency and consistency tended to improve more in the phloroglucinol group than placebo group, but there were no statistical significances. The IBS-QOL also showed a trend towards a better efficacy in the phloroglucinol group than placebo group without statistical significances. Further large scaled studies are warranted to investigate the efficacy of phloroglucinol on bowel habits and QOL.

Regarding safety, some antispasmodics, such as anticholinergics, are associated with anticholinergic side effects, including dry mouth, glaucoma, blurred vision, urinary retention, and tachycardia, which limit their use. Phloroglucinol is not associated with these anticholinergic side effects; a previous study reported that few patients experienced adverse effects such as mild headaches and allergic reactions. ${ }^{35}$ In addition, a French study showed that the phloroglucinol did not have a teratogenic effect in pregnant women. ${ }^{41}$ In our study, only 2 patients complained of mild nausea, which was not serious enough to discontinue the study drug. Therefore, the therapeutic use of phloroglucinol raised no safety concerns.

There were several limitations in this study. First, the duration of our study was relatively short ( 3 weeks). Because IBS is a chronic and recurrent disease, short-term clinical trials to confirm if a treatment is effective in improving IBS are considered to have limited clinical relevance. ${ }^{42}$ However, antispasmodics are drugs that are used to temporally relieve IBS symptoms rather than to target longterm control, and thus short-term study may be enough. Actually, in many clinical trials for IBS, drug efficacies were observed in for week or 2 weeks. ${ }^{33,43}$ Second, this study did not consider diet. Several studies have shown that certain foods play an important role in the development and exacerbation of IBS symptoms in a majority of IBS patients. ${ }^{44,45}$ Instead of controlling diet, we instructed patients to continue consuming their usual diet throughout the study period. Third, a relatively small sample size can lead to bias in the study re- sults, as previously mentioned. However, to the best of our knowledge, this is the first randomized, double-blind, placebo-controlled study to evaluate overall and individual symptoms as well as stool parameters in patients with IBS-D treated with phloroglucinol. Additionally, we confirmed that the degree of anxiety and depression was not different between the 2 groups, thus excluding the effects of psychological distress on the effect of study treatment.

In conclusion, phloroglucinol can be effective and safe for the management of overall symptoms in patients with IBS-D. Stool frequency and QOL tend to improve by phloroglucinol. Further studies are needed to validate the efficacy of phloroglucinol in a larger sample size and long-term period.

Financial support: This work was supported by a grant from the National Research Foundation of Korea (NRF) funded by the Korea government (MEST) (No. 2017R1D1A1B03031924) and by research funding from DAE HWA Pharmaceutical Co, Ltd, Gangwon-do, Korea.

\section{Conflicts of interest: None.}

Author contributions: Seung Yong Shin and Bong Ki Cha substantially contributed to conception, design, analysis, interpretation of data, drafting the article, and revising it critically for important content; Chang Hwan Choi substantially contributed to conception and design, final approval of the version to be published, and agreement to be accountable for all aspects of the work; WonSeok Kim, Jae Yong Park, and Jeong Wook Kim prepared and revised the article critically for important intellectual content; and all authors reviewed the manuscript.

\section{References}

1. Mearin F, Lacy BE, Chang L, et al. Bowel disorders. Gastroenterology Published Online First: 18 Feb 2016. doi: 10.1053/j.gastro.2016.02.031.

2. Gwee KA, Gonlachanvit S, Ghoshal UC, et al. Second Asian Consensus on irritable bowel syndrome. J Neurogastroenterol Motil 2019;25:343362 .

3. Drossman DA, Camilleri M, Mayer EA, Whitehead WE. AGA technical review on irritable bowel syndrome. Gastroenterology 2002;123:21082131.

4. Ford AC, Lacy BE, Talley NJ. Irritable bowel syndrome. N Engl J Med 2017;376:2566-2578.

5. Lee YJ, Park KS. Irritable bowel syndrome: emerging paradigm in pathophysiology. World J Gastroenterol 2014;20:2456-2469.

6. Fukui $\mathrm{H}, \mathrm{Xu} \mathrm{X}$, Miwa $\mathrm{H}$. Role of gut microbiota-gut hormone axis in 
the pathophysiology of functional gastrointestinal disorders. J Neurogastroenterol Motil 2018;24:367-386.

7. Kanazawa M, Palsson OS, Thiwan SI, et al. Contributions of pain sensitivity and colonic motility to IBS symptom severity and predominant bowel habits. Am J Gastroenterol 2008;103:2550-2561.

8. Brandt LJ, Chey WD, Foxx-Orenstein AE, et al. An evidence-based position statement on the management of irritable bowel syndrome. Am J Gastroenterol 2009;104(suppl 1):S1-S35.

9. Gorard DA, Libby GW, Farthing MJ. Ambulatory small intestinal motility in 'diarrhoea' predominant irritable bowel syndrome. Gut 1994;35:203-210.

10. Kellow JE, Phillips SF. Altered small bowel motility in irritable bowel syndrome is correlated with symptoms. Gastroenterology 1987;92:18851893.

11. Kellow JE, Phillips SF, Miller LJ, Zinsmeister AR. Dysmotility of the small intestine in irritable bowel syndrome. Gut 1988;29:1236-1243.

12. Chey WY, Jin HO, Lee MH, Sun SW, Lee KY. Colonic motility abnormality in patients with irritable bowel syndrome exhibiting abdominal pain and diarrhea. Am J Gastroenterol 2001;96:1499-1506.

13. Chey WD, Maneerattaporn M, Saad R. Pharmacologic and complementary and alternative medicine therapies for irritable bowel syndrome. Gut Liver 2011;5:253-266.

14. Ford AC, Talley NJ, Spiegel BM, et al. Effect of fibre, antispasmodics, and peppermint oil in the treatment of irritable bowel syndrome: systematic review and meta-analysis. BMJ 2008;337:a2313.

15. Ruepert L, Quartero AO, de Wit NJ, van der Heijden GJ, Rubin G, Muris JW. Bulking agents, antispasmodics and antidepressants for the treatment of irritable bowel syndrome. Cochrane Database Syst Rev 2011:CD003460.

16. Lesbros-Pantoflickova D, Michetti P, Fried M, Beglinger C, Blum AL. Meta-analysis: the treatment of irritable bowel syndrome. Aliment Pharmacol Ther 2004;20:1253-1269.

17. Tack J, Fried M, Houghton LA, Spicak J, Fisher G. Systematic review: the efficacy of treatments for irritable bowel syndrome--a European perspective. Aliment Pharmacol Ther 2006;24:183-205.

18. Martínez-Vázquez MA, Vázquez-Elizondo G, González-González JA, Gutiérrez-Udave R, Maldonado-Garza HJ, Bosques-Padilla FJ. Effect of antispasmodic agents, alone or in combination, in the treatment of irritable bowel syndrome: systematic review and meta-analysis. Rev Gastroenterol Mex 2012;77:82-90.

19. Bueno L, Fioramonti J, Delvaux M, Frexinos J. Mediators and pharmacology of visceral sensitivity: from basic to clinical investigations. Gastroenterology 1997;112:1714-1743.

20. Lazaraki G, Chatzimavroudis G, Katsinelos P. Recent advances in pharmacological treatment of irritable bowel syndrome. World J Gastroenterol 2014;20:8867-8885.

21. Cargill G, Salin B, Lubin S, Kohler F, Coste T, Rautureau J. [Effect of phloroglucinol on rectosigmoid motility stimulated by a test meal. Study in patients with irritable bowel syndrome.] Presse Med 1992;21:19-23. [French]

22. Louvel D, Delvaux M, Staumont G, et al. Intracolonic injection of glycerol: a model for abdominal pain in irritable bowel syndrome? Gastroen- terology 1996;110:351-361.

23. Veldhuyzen van Zanten SJ, Talley NJ, Bytzer P, Klein KB, Whorwell PJ, Zinsmeister AR. Design of treatment trials for functional gastrointestinal disorders. Gut 1999;45(suppl 2):II69-II77.

24. Heaton KW, O’Donnell LJ. An office guide to whole-gut transit time. Patients' recollection of their stool form. J Clin Gastroenterol 1994;19:2830.

25. Drossman DA, Patrick DL, Whitehead WE, et al. Further validation of the IBS-QOL: a disease-specific quality-of-life questionnaire. Am J Gastroenterol 2000;95:999-1007.

26. Zigmond AS, Snaith RP. The hospital anxiety and depression scale. Acta Psychiatr Scand 1983;67:361-370

27. Oh SM, Min KJ, Park DB. [A study on the standardization of the hospital anxiety and depression scale for Koreans: a comparison of normal, depressed and anxious gourps.] J Korean Neuropsychiatr Assoc 1999;38:289-296. [Korean]

28. Kwon JG, Park KS, Park JH, et al. [Guidelines for the treatment of irritable bowel syndrome.] Korean J Gastroenterol 2011;57:82-99. [Korean]

29. Boeckxstaens G, Corazziari ES, Mearin F, Tack J. IBS and the role of otilonium bromide. Int J Colorectal Dis 2013;28:295-304.

30. Poynard T, Regimbeau C, Benhamou Y. Meta-analysis of smooth muscle relaxants in the treatment of irritable bowel syndrome. Aliment Pharmacol Ther 2001;15:355-361.

31. Vanuytsel T, Tack JF, Boeckxstaens GE. Treatment of abdominal pain in irritable bowel syndrome. J Gastroenterol 2014;49:1193-1205.

32. Clavé P, Acalovschi M, Triantafillidis JK, et al. Randomised clinical trial: otilonium bromide improves frequency of abdominal pain, severity of distention and time to relapse in patients with irritable bowel syndrome. Aliment Pharmacol Ther 2011;34:432-442.

33. Lee KN, Lee OY, Choi MG, et al. Efficacy and safety of tiropramide in the treatment of patients with irritable bowel syndrome: a multicenter, randomized, double-blind, non-inferiority trial, compared with octylonium. J Neurogastroenterol Motil 2014;20:113-121.

34. Chassany O, Bonaz B, Bruley DES Varannes S, et al. Acute exacerbation of pain in irritable bowel syndrome: efficacy of phloroglucinol/trimethylphloroglucinol. A randomized, double-blind, placebo-controlled study. Aliment Pharmacol Ther 2007;25:1115-1123.

35. Jafri W, Yakoob J, Hussain S, Jafri N, Islam M. Phloroglucinol in irritable bowel syndrome. J Pak Med Assoc 2006;56:5-8.

36. Patel SM, Stason WB, Legedza A, et al. The placebo effect in irritable bowel syndrome trials: a meta-analysis. Neurogastroenterol Motil 2005;17:332-340.

37. Thompson WG. Placebos: a review of the placebo response. Am J Gastroenterol 2000;95:1637-1643.

38. Cha BK, Jung SM, Choi CH, et al. The effect of a multispecies probiotic mixture on the symptoms and fecal microbiota in diarrhea-dominant irritable bowel syndrome: a randomized, double-blind, placebo-controlled trial. J Clin Gastroenterol 2012;46:220-227

39. Choi CH, Kwon JG, Kim SK, et al. Efficacy of combination therapy with probiotics and mosapride in patients with IBS without diarrhea: a randomized, double-blind, placebo-controlled, multicenter, phase II trial. Neurogastroenterol Motil 2015;27:705-716. 
40. Choi CH, Jo SY, Park HJ, Chang SK, Byeon JS, Myung SJ. A randomized, double-blind, placebo-controlled multicenter trial of saccharomyces boulardii in irritable bowel syndrome: effect on quality of life. J Clin Gastroenterol 2011;45:679-683.

41. Lacroix I, Hurault-Delarue C, Kessler S, et al. [First epidemiologic data about phloroglucinol exposure during first trimester of pregnancy.] Gynecol Obstet Fertil 2011;39:694-697. [French]

42. Akehurst R, Kaltenthaler E. Treatment of irritable bowel syndrome: a review of randomised controlled trials. Gut 2001;48:272-282.

43. Fukudo S, Kinoshita Y, Okumura T, et al. Ramosetron reduces symp- toms of irritable bowel syndrome with diarrhea and improves quality of life in women. Gastroenterology 2016;150:358-366, e8.

44. Staudacher HM, Whelan K. The low FODMAP diet: recent advances in understanding its mechanisms and efficacy in IBS. Gut 2017;66:15171527.

45. Varjú P, Farkas N, Hegyi P, et al. Low fermentable oligosaccharides, disaccharides, monosaccharides and polyols (FODMAP) diet improves symptoms in adults suffering from irritable bowel syndrome (IBS) compared to standard IBS diet: a meta-analysis of clinical studies. PLoS One 2017;12:e0182942. 